\title{
PERFIL VOCAL DE REGENTES DE CORAL DO ESTADO DE SÃO PAULO
}

\section{Vocal profile of choir conductors in the State of São Paulo}

\author{
Maria Inês Beltrati Cornacchioni Rehder (1), Mara Suzana Behlau (2)
}

\begin{abstract}
RESUMO
Objetivo: traçar o perfil vocal de regentes de corais do Estado de São Paulo. Métodos: participaram deste estudo 150 regentes de corais do Estado de São Paulo. A coleta foi feita individualmente pelo mesmo avaliador, nos locais de trabalho dos entrevistados, através de questionário fechado. Resultados: os regentes tem em média 8,4 anos de exercício da profissão, regem 1 coral e já fizeram de 1 a 5 anos de aula de canto. Ensaiam os naipes em separado, cantam junto com os naipes, fazem aquecimento e não fazem desaquecimento vocal. Para a afinação do coral, usam piano e/ou a própria voz. A maior parte considera a voz falada igual a cantada e ambas eficientes, há concordância no que se refere ao dom e a técnica como componentes necessários a uma boa voz cantada. Com os anos de exercício de regência, a tessitura vocal está mais ampla, a qualidade mais estável, a passagem mais controlada e a intensidade no pianíssimo e no fortíssimo permaneceram inalteradas. As queixas vocais mais apontadas foram: pigarro, rouquidão, garganta seca, acúmulo de secreção na garganta, cansaço após fala, cansaço após canto e tensão na garganta. A maior parte dos regentes apresenta até 3 sintomas vocais, não fumam, não bebem, não costumam gritar, não pigarreiam, falam muito e comem tarde da noite. Conclusão: houve associação de sintomas vocais com piora e/ou restrição de diversos parâmetros específicos da voz cantada. O parâmetro que mais se alterou frente aos sintomas vocais foi a qualidade vocal.
\end{abstract}

DESCRITORES: Voz; Qualidade da Voz; Treinamento da Voz; Música; Distúrbios da Voz

\section{INTRODUÇÃO}

Reger é o ato de transmitir a um conjunto instrumental ou vocal, por meio de gestos convencionais, o conteúdo rítmico e expressivo de uma obra musical. O regente é o elemento decisivo entre o simbólico triângulo formado pela obra, os executantes e os ouvintes, transformando a sonoridade em uma realidade efetiva. Cabe ao regente conciliar e harmonizar as vozes dentro do grupo coral, buscando uma identidade sonora única sem discrepâncias, com qualidade artística equilibrada ${ }^{1-3}$. A arte de dirigir um coro exige requisitos de cultura geral e qualidades especiais de liderança. Exige ainda,

(1) Fonoaudióloga; Clínica Parole de Fonoaudiologia; Doutora em Distúrbios da Comunicação Humana pela Universidade Federal de São Paulo.

(2) Fonoaudióloga; Centro de Estudos da Voz; Doutora em Distúrbios da Comunicação Humana pela Universidade Federal de São Paulo. grande preparação musical e técnica, incluindo conhecimento amplo da voz sob os pontos de vista acústico, fisiológico e psicológico ${ }^{4,5}$.

O regente dispõe de três meios básicos de expressão para comunicar-se com o conjunto: o gesto, a mímica e a voz ${ }^{6,7}$. É grande sua responsabilidade no que diz respeito a sua própria performance vocal, uma vez que esta tende ser imitada pelo grupo ${ }^{4,5}$. O regente ocupa uma posição de alto risco no que diz respeito ao desenvolvimento de um problema de voz ou de laringe. O uso excessivo da voz falada e cantada é uma realidade durante os ensaios, o que é, ainda, acentuado pela competição sonora com o próprio grupo vocal. É comum que o regente tente sobrepujar o volume do coro para se fazer ouvir $6,8,9$.

Pesquisas relacionadas à voz cantada tem recebido recentemente o interesse de profissionais de diversas áreas, porém pouco se sabe ainda sobre a dinâmica profissional e especificidades inerentes a profissão de regente de coral, incluindo os aspectos vocais. 
O objetivo deste estudo é traçar o perfil vocal de regentes de coral do Estado de São Paulo, com o intuíto de conhecer aspectos particulares deste profissional tão importante na vida sócio-cultural e na saúde do país.

\section{MÉTODOS}

Este foi um estudo transversal do qual participaram 150 sujeitos voluntários, 95 (63,3\%) do sexo feminino e $55(36,6 \%)$ do sexo masculino, na faixa etária entre 20 e 65 anos de idade, com idade média de 45,3 anos de idade, todos regentes de um ou mais corais do Estado de São Paulo.

Para a seleção dos sujeitos foram incluídos regentes de corais infantis, juvenis e adultos, com ou sem formação acadêmica específica, de ambos os sexos. O critério de inclusão foi o de estar atuando na função regente na data da coleta de dados.

A coleta de dados foi realizada individualmente pelo mesmo avaliador, nos locais de trabalho dos entrevistados, através de questionário fechado ${ }^{8}$ (Figura 1) incluindo os seguintes aspectos:

- composição e organização do(s) coral(is) que rege quanto ao número de participantes, faixa etária, sexo, tipo (amador ou profissional) e divisão de naipes;

- anos de exercício de regência;

- caracterização pessoal do regente: número de corais que rege, classificação vocal e prática de aulas de canto;

- procedimentos de atuação junto ao(s) coral(is) no que se refere a: ensaio de naipes, cantar ou não junto com os naipes, recursos utilizados para a afinação e prática de aquecimento e desaquecimento vocal;

- auto avaliação do regente quanto a: qualidade da voz falada em relação à cantada, eficiência das vozes falada e cantada e análise do fator básico para uma boa voz cantada;

- comparação temporal da voz entre o período inicial e atual da regência relacionada a: tessitura, intensidade no pianíssimo, intensidade no fortíssimo, qualidade vocal, passagem e afinação;

- queixas e sintomas vocais: rouquidão, pigarro, tosse, acúmulo de secreção na garganta, dor ao falar, dor ao cantar, cansaço após fala, cansaço após canto, ardor na garganta, tensão na garganta, perda de voz com o uso e garganta seca; hábitos: fumo, álcool, falar muito, falar em intensidade forte, gritar, pigarrear e comer tarde da noite.

Na maior parte das questões, era cabível apenas uma resposta que deveria ser assinalada com um xis. As questões relacionadas a afinação do coral, queixas e sintomas vocais e hábitos poderiam receber mais de uma resposta por se tratarem de ítens coexistentes e não excludentes. Nas questões relacionadas a hábitos, foram incluídas três possibilidades: presença (sim), ausência (não) e ocorrência rara (as vezes) para caracterizar melhor a presença dos hábitos selecionados. Os dados coletados através do questionário foram compilados de forma descritiva e apresentados nas Tabelas 1 a 7, 11 e 12.

Os dados relacionados aos procedimentos de atuação junto aos corais e comparação temporal da voz foram confrontados individualmente com queixas e sintomas vocais e hábitos a fim de se obter um perfil vocal individual de cada regente. Os perfis vocais individuais foram, então, confrontados entre si possibilitando o traçado do perfil vocal do grupo. Estes dados encontram-se nas Tabelas de 8, 9 e 10.

Esta pesquisa foi aprovada pela Comissão de Ética em Pesquisa da Universidade Federal de São Paulo, sob o número 1167/01.

Para o tratamento estatístico foram utilizados os teste de qui-quadrado para verificar diferenças nas atribuições de uma característica em função de outra, e o grau de relacionamento entre duas características categorizadas. Em casos de baixa freqüência, onde o teste de qui-quadrado perde a eficiência, utilizou-se o teste alternativo de Fischer. O nível de significância utilizado nos testes foi de $5 \%$.

\section{RESULTADOS}

Embora se tenha entrevistado 150 regentes de coral, uma parte deste contingente rege mais de um grupo, o que possibilitou ter informações sobre mais de 281 corais do Estado de São Paulo. Esta amostra permite colocar que os corais do Estado de São Paulo são mistos, compostos em sua maioria por 20 a 40 participantes e por indivíduos adultos. A quase totalidade dos corais dessa amostra 275 $(97,9 \%)$ são amadores, divididos em sua maioria em duas vozes (Tabela 1).

Com relação a caracterização do regente, foi possível observar que em sua maioria, $83(55,3 \%)$, rege 1 coral, uma parte desses indivíduos, 80 (53,3\%), já fez aulas de canto e dos que fizeram ou fazem, 131 $(87,3 \%)$ mantiveram-na de 1 a 5 anos $(82,4 \%)$. Com relação à classificação vocal dos regentes do sexo feminino, 39 (41,1\%) são sopranos, 37 (38,9\%) são meio-sopranos e 19 (20\%) são contraltos. A classificação vocal dos regentes do sexo masculino apontou $26(47,3 \%)$ de tenores, $23(41,8 \%)$ de barítonos e $6(10,9 \%)$ de baixos (Tabela 2$)$.

Os regentes foram bastante coicidentes em suas respostas com relação aos procedimentos de atuação junto aos corais: a maioria, 96 (64\%), ensaia os naipes em separado, $146(97,3 \%)$ cantam junto com os naipes, $140(93,3 \%)$ fazem aquecimento 
Nome: Data de nascimento:

Formação:

Local e ano de formação:

Quantos anos tem de exercício de regência?

Quantos corais você rege atualmente?

\begin{tabular}{|l|l|}
\hline & 1 \\
\hline & 2 \\
\hline & 3 \\
\hline & 4 \\
\hline & mais de 4 \\
\hline
\end{tabular}

Coral onde exerce a regência

Sobre o coral

№ de participantes

\begin{tabular}{|l|l|}
\hline & até 20 \\
\hline & de 20 a 40 \\
\hline & mais de 40 \\
\hline
\end{tabular}

\section{Classificação}

\begin{tabular}{|l|l|}
\hline & infantil \\
\hline & juvenil \\
\hline & adulto \\
\hline
\end{tabular}

\begin{tabular}{|l|l|}
\hline & masculino \\
\hline & feminino \\
\hline & misto \\
\hline
\end{tabular}

\begin{tabular}{|l|l|}
\hline & amador \\
\hline & profissional \\
\hline
\end{tabular}

№ de naipes

\begin{tabular}{|l|l|}
\hline & 2 \\
\hline & 4 \\
\hline & 6 \\
\hline & não há divisão \\
\hline
\end{tabular}

Você ensaia os naipes em separado, antes do ensaio com o grupo todo? ( ) sim ( ) não

Durante os ensaios você costuma cantar junto com os diferentes naipes? ( ) sim ( ) não

\section{Para a afinação do coral você usa:}

\begin{tabular}{|l|l|}
\hline & diapasão \\
\hline & escaleta \\
\hline & piano \\
\hline & sua própria voz \\
\hline
\end{tabular}

Com relação a aulas de canto, você: ( ) nunca fez ( ) já fez ( ) faz

№ de anos de aula de canto: Para você, a voz falada é: Para você, a voz cantada é:

\begin{tabular}{|l|l|}
\hline & de 1 a 5 anos \\
\hline & de 5 a 10 anos \\
\hline & mais de 10 anos \\
\hline
\end{tabular}

Para você, a voz cantada é:
\begin{tabular}{|l|l|}
\hline & dom \\
\hline & técnica \\
\hline & ambos
\end{tabular}

A sua voz falada é eficiente? ( ) sim ( ) não

A sua voz cantada é eficiente? ( ) sim ( ) não 
Qual é a sua classificação vocal:

\begin{tabular}{|l|l|l|l|}
\hline & soprano & & tenor \\
\hline & mezzo & & barítono \\
\hline & contralto & & baixo \\
\hline & outra: & \multicolumn{2}{|c|}{} \\
\cline { 2 - 3 }
\end{tabular}

Com os passar dos anos, atuando como regente você acha que sua voz está, co

\begin{tabular}{|l|l|}
\hline & mais ampla \\
\hline & mais restrita \\
\hline & mesma tessitura \\
\hline
\end{tabular}

com relação a intensidade

\begin{tabular}{|l|l|}
\hline & melhor no pianíssimo \\
\hline & pior no pianíssimo \\
\hline & igual no pianíssimo \\
\hline
\end{tabular}

\begin{tabular}{|l|l|}
\hline & melhor no fortíssimo \\
\hline & pior no fortíssimo \\
\hline & igual no fortíssimo \\
\hline
\end{tabular}

com relação a qualidade

\begin{tabular}{|l|l|}
\hline & mais estável \\
\hline & menos estável \\
\hline & mesma qualidade \\
\hline
\end{tabular}
com relação a passagem

\begin{tabular}{|l|l|}
\hline & melhor \\
\hline & pior \\
\hline & igual \\
\hline
\end{tabular}
com relação a afinação

Você apresenta algum dos sintomas abaixo relacionados?

\begin{tabular}{|l|l|}
\hline & rouquidão \\
\hline & pigarro \\
\hline & tosse \\
\hline & acúmulo de secreção na garganta \\
\hline & dor ao falar \\
\hline & dor ao cantar \\
\hline & cansaço após fala \\
\hline & cansaço após canto \\
\hline & ardor na garganta \\
\hline & tensão na garganta \\
\hline & perda de voz com o uso \\
\hline & garganta seca \\
\hline
\end{tabular}

Você:

\begin{tabular}{|l|l|l|l|}
\hline & Sim & Não & $\begin{array}{c}\text { Ás } \\
\text { vezes }\end{array}$ \\
\hline Fuma & & & \\
\hline Bebe & & & \\
\hline Fala muito & & & \\
\hline $\begin{array}{l}\text { Fala em intensidade } \\
\text { forte }\end{array}$ & & & \\
\hline Grita & & & \\
\hline Pigarreia & & & \\
\hline Come tarde da noite & & & \\
\hline
\end{tabular}

Antes dos ensaios e apresentações você faz aquecimento vocal? （ ) sim （ ) não

Depois dos ensaios e apresentações você faz desaquecimento vocal? ( ) sim ( ) não 
Tabela 1 - Distribuição numérica e percentual da composição e organização dos corais

\begin{tabular}{|c|c|c|}
\hline $\begin{array}{l}\text { Composição e } \\
\text { organização }\end{array}$ & $\mathbf{n}$ & $\%$ \\
\hline \multicolumn{3}{|c|}{ Número de participantes } \\
\hline-120 & 81 & 28,8 \\
\hline$|-| 40$ & 126 & 44,8 \\
\hline $1-40$ & 74 & 26,3 \\
\hline TOTAL & 281 & 100,0 \\
\hline \multicolumn{3}{|c|}{ Faixa etária dos participantes } \\
\hline infantil & 84 & 29,9 \\
\hline juvenil & 48 & 17,1 \\
\hline adulto & 149 & 53,0 \\
\hline TOTAL & 281 & 100,0 \\
\hline \multicolumn{3}{|l|}{ Sexo dos participantes } \\
\hline feminino & 11 & 3,9 \\
\hline masculino & 0 & 0,0 \\
\hline misto & 270 & 96,1 \\
\hline TOTAL & 281 & 100,0 \\
\hline \multicolumn{3}{|l|}{ Tipo de coral } \\
\hline amador & 275 & 97,9 \\
\hline profissional & 6 & 2,1 \\
\hline TOTAL & 281 & 100,0 \\
\hline \multicolumn{3}{|c|}{ Divisão de naipes - infantil } \\
\hline 2 & 46 & 54,8 \\
\hline 3 & 2 & 2,4 \\
\hline 4 & 1 & 1,2 \\
\hline Não há divisão & 35 & 41,7 \\
\hline TOTAL & 84 & 100,0 \\
\hline \multicolumn{3}{|c|}{ Divisão de naipes - juvenil } \\
\hline 2 & 22 & 45,8 \\
\hline 3 & 2 & 4,2 \\
\hline 4 & 15 & 31,2 \\
\hline Não há divisão & 9 & 18,8 \\
\hline TOTAL & 48 & 100,0 \\
\hline \multicolumn{3}{|c|}{ Divisão de naipes - adulto } \\
\hline 2 & 25 & 16,8 \\
\hline 3 & 3 & 2,0 \\
\hline 4 & 112 & 75,2 \\
\hline 6 & 6 & 4,0 \\
\hline 8 & 2 & 1,3 \\
\hline Não há divisão & 1 & 0,7 \\
\hline TOTAL & 149 & 100,0 \\
\hline
\end{tabular}

vocal e $133(89,3 \%)$ não fazem desaquecimento vocal (Tabela 3$)$.

O piano e a própria voz foram os recursos mais apontados, $82,4 \%$ para a afinação do coral (Tabela 4).
Tabela 2 - Distribuição numérica e percentual da caracterização do regente

\begin{tabular}{|c|c|c|}
\hline $\begin{array}{l}\text { Caracterização } \\
\text { do regente }\end{array}$ & $\mathbf{n}$ & $\%$ \\
\hline \multicolumn{3}{|c|}{ Números de corais que rege } \\
\hline 1 & 83 & 55,3 \\
\hline 2 & 34 & 22,7 \\
\hline 3 & 15 & 10,0 \\
\hline 4 & 9 & 6,0 \\
\hline mais de 4 & 9 & 6,0 \\
\hline TOTAL & 150 & 100,0 \\
\hline \multicolumn{3}{|l|}{ Classificação vocal } \\
\hline soprano & 39 & 41,1 \\
\hline meio-soprano & 37 & 38,9 \\
\hline contralto & 19 & 20,0 \\
\hline TOTAL & 95 & 100,0 \\
\hline \multicolumn{3}{|l|}{ Classificação vocal } \\
\hline tenor & 26 & 47,3 \\
\hline barítono & 23 & 41,8 \\
\hline baixo & 6 & 10,9 \\
\hline TOTAL & 55 & 100,0 \\
\hline \multicolumn{3}{|c|}{ Prática de aulas de canto } \\
\hline nunca fez & 19 & 12,7 \\
\hline já fez & 80 & 53,3 \\
\hline faz & 51 & 34,0 \\
\hline TOTAL & 150 & 100,0 \\
\hline \multicolumn{3}{|c|}{ Número de anos de aula de canto } \\
\hline de 1 a 5 anos & 108 & 82,4 \\
\hline de 5 a 10 anos & 17 & 13,0 \\
\hline mais de 10 anos & 6 & 4,6 \\
\hline TOTAL & 131 & 100,0 \\
\hline
\end{tabular}

Tabela 3 - Distribuição numérica e percentual dos procedimentos de atuação dos regentes junto ao(s) coral(is)

\begin{tabular}{|c|c|c|}
\hline Procedimentos & $\mathbf{n}$ & $\%$ \\
\hline \multicolumn{3}{|c|}{ Ensaio de naipes em separado } \\
\hline $\operatorname{sim}$ & 96 & 64,0 \\
\hline não & 54 & 36,0 \\
\hline \multicolumn{3}{|c|}{ Cantar junto com os naipes } \\
\hline $\operatorname{sim}$ & 146 & 97,3 \\
\hline não & 4 & 2,7 \\
\hline \multicolumn{3}{|c|}{ Aquecimento vocal } \\
\hline $\operatorname{sim}$ & 140 & 93,3 \\
\hline não & 10 & 6,7 \\
\hline \multicolumn{3}{|c|}{ Desaquecimento vocal } \\
\hline $\operatorname{sim}$ & 16 & 10,7 \\
\hline não & 133 & 89,3 \\
\hline
\end{tabular}


Tabela 4 - Recursos utilizados pelo regente para a afinação do(s) coral(is) que rege

\begin{tabular}{lrr}
\hline Recursos & $\mathbf{n}$ & $\%$ \\
\hline Diapasão & 49 & 17,2 \\
Escaleta & 1 & 0,4 \\
Piano & 136 & 47,7 \\
A própria voz & 99 & 34,7 \\
\hline
\end{tabular}

Com relação a auto-avaliação, a maior parte dos regentes da amostra, 93 sujeitos (62,0\%), consideraram a qualidade da voz falada igual a qualidade da voz cantada, 132 (88\%) consideraram a voz falada eficiente e 129 (86\%) consideraram a voz cantada eficiente. Na questão referente ao componete básico para uma boa voz cantada, dom e/ou técnica, obteve dom e técnica em $76,7 \%$ das respostas (Tabela 5).

Tabela 5 - Distribuição numérica e percentual da auto-avaliação dos regentes

\begin{tabular}{lrr}
\hline $\begin{array}{l}\text { Auto avaliação voz } \\
\text { falada x voz cantada }\end{array}$ & $\mathbf{n}$ & $\%$ \\
\hline $\begin{array}{l}\text { Qualidade } \\
\text { melhor }\end{array}$ & 16 & 10,7 \\
$\quad$ pior & 41 & 27,3 \\
$\quad$ igual & 93 & 62,0 \\
Voz falada eficiente & & \\
$\quad$ sim & 132 & 88,0 \\
não & 18 & 12,0 \\
Voz cantada eficiente & & \\
$\quad$ sim & 129 & 86,0 \\
$\quad$ não & 21 & 14,0 \\
Voz cantada & & \\
$\quad$ dom & 2 & 1,3 \\
técnica & 33 & 22,0 \\
ambos & 115 & 76,7 \\
\hline
\end{tabular}

$\mathrm{Na}$ comparação de aspectos vocais entre os períodos inicial e atual da regência, observa-se os seguintes dados: $97(64,7 \%)$ consideraram que a tessitura está mais ampla, 81 (54\%) que a qualidade vocal está mais estável, $78(52 \%)$ que a passagem está mais controlada e $70(46,7 \%)$ que a afinação está melhor. Quando solicitados a comparar a intensidade no pianíssimo e a intensidade no fortíssimo, entre os períodos inicial e atual da regência, $60(40 \%)$ consideraram o pianíssimo igual e $59(39,3 \%)$ consideraram-no melhor; $70(46,7 \%)$ referiram melhora no fortíssimo e $63(42,0 \%)$ referiram igualdade (Tabela 6 ).

As queixas e sintomas vocais apontados pelos regentes forma: $47(31,3 \%)$ apontaram pigarro, $39(26 \%)$ rouquidão, $39(26 \%)$ garganta seca, 37 $(24,7 \%)$ acúmulo de secreção na garganta, 37 $(24,7 \%)$ cansaço após fala, $36(24 \%)$ cansaço após canto, $31(20,7 \%)$ tensão na garganta, $27(18 \%)$ perda de voz com o uso, $22(14,7 \%)$ tosse, 13 $(8,7 \%)$ ardor na garganta, $12(8 \%)$ dor ao cantar e $10(6,7 \%)$ dor ao falar (Tabela 7$)$.

Ao verificar o grau de relacionamento entre duas características categorizadas, obteve-se significância estatística na associação de voz rouca a uma tessitura mais restrita, a uma pior intensidade no pianíssimo, a uma voz menos estável e a uma passagem pior; associação de garganta seca com tessitura mais restrita; associação de acúmulo de secreção na garganta a uma voz falada menos eficiente, a pior intensidade no pianíssimo; a uma voz menos estável e a uma pior passagem (Tabela 8). Observa-se a associação de cansaço após fala com uma pior intensidade no pianíssimo e o cansaço após canto à pratica de ensaio dos naipes em separado; a uma voz falada melhor que a cantada e a não eficiência da voz cantada (Tabela 9). Observase ainda a associação do parâmetro tensão na garganta a uma pior intensidade no pianíssimo e a uma qualidade vocal menos estável (Tabela 10).

$\mathrm{Na}$ comparação da quantidade de queixas e sintomas vocais com o número de regentes foi

Tabela 6 - Distribuição numérica e percentual da comparação temporal de aspectos vocais entre o período inicial e atual da regência

\begin{tabular}{lcccccc}
\hline \multirow{2}{*}{ Comparação } & \multicolumn{2}{c}{$+\mathbf{n}$} & $\mathbf{3}$ & \multicolumn{1}{c}{-} \\
\cline { 2 - 7 } & $\mathbf{n}$ & $\%$ & $\mathbf{n}$ & $\%$ & $\mathbf{n}$ & $\%$ \\
\hline Tessitura & 97 & 64,7 & 29 & 19,3 & 24 & 16,0 \\
Intensidade no pianíssimo & 59 & 39,3 & 60 & 40,0 & 31 & 20,7 \\
Intensidade no fortíssimo & 70 & 46,7 & 63 & 42,0 & 17 & 11,3 \\
Qualidade vocal & 81 & 54,0 & 32 & 21,3 & 37 & 24,7 \\
Passagem & 78 & 52,0 & 57 & 38,0 & 15 & 10,0 \\
Afinação & 70 & 46,7 & 63 & 42,0 & 17 & 11,3 \\
\hline
\end{tabular}

LEGENDA: + mais ampla, melhor, mais estável; = mesma , igual; - mais restrita, pior, menos estável 
Tabela 7 - Distribuição numérica e percentual de queixas e sintomas vocais apresentados pelos regentes

\begin{tabular}{lcc}
\hline Sinais e sintomas & $\mathbf{n}$ & $\%$ \\
\hline Pigarro & 47 & 31,3 \\
Rouquidão & 39 & 26,0 \\
Garganta seca & 39 & 26,0 \\
Acúmulo de secreção na garganta & 37 & 24,7 \\
Cansaço após fala & 37 & 24,7 \\
Cansaço após canto & 36 & 24,0 \\
Tensão na garganta & 31 & 20,7 \\
Perda de voz com o uso & 27 & 18,0 \\
Tosse & 22 & 14,7 \\
Ardor na garganta & 13 & 8,7 \\
Dor ao cantar & 12 & 8,0 \\
Dor ao falar & 10 & 6,7 \\
\hline
\end{tabular}

Tabela 8 - Análise comparativa do grau de relacionamento entre as queixas de pigarro e rouquidão e parâmetros de voz falada e cantada

\begin{tabular}{lcc}
\hline Parâmetros & $\begin{array}{c}\text { Valor } \\
\text { crítico }\end{array}$ & $\begin{array}{c}\text { Valor } \\
\text { observado }\end{array}$ \\
\hline Pigarro x eficiência da voz falada & 3,84 & 1,63 \\
Pigarro x eficiência da voz cantada & 3,84 & 0,09 \\
Pigarro x comparação temporal da intensidade no pianíssimo & 5,99 & 2,58 \\
Pigarro x compação temporal da intensidade no fortíssimo & 5,99 & 0,14 \\
Pigarro x comparação temporal da qualidade vocal & 5,99 & 4,24 \\
Pigarro x fala em intensidade forte & 5,99 & 0,36 \\
Rouquidão x eficiência da voz cantada & 3,84 & 0,68 \\
Rouquidão x comparação temporal da tessitura vocal & 5,99 & $15,89^{*}$ \\
Rouquidão x compação temporal da intensidade no pianíssimo & 5,99 & $7,52^{*}$ \\
Rouquidão x comparação temporal da intensidade no fortíssimo & 5,99 & 0,24 \\
Rouquidão x compação temporal da qualidade vocal & 5,99 & $17,64^{*}$ \\
Rouquidão x compação temporal da passagem & 5,99 & $10,87^{*}$ \\
Garganta seca x ensaio de naipes em separado & 3,84 & 0,63 \\
Garganta seca x voz falada em relação a cantada & 5,99 & 1,89 \\
Garganta seca x voz cantada eficiente & 3,84 & 0,68 \\
Garganta seca x comparação temporal da tessitura vocal & 5,99 & $7,23^{*}$ \\
Garganta seca x comparação temporal da intensidade vocal no pianíssimo & 5,99 & 0,80 \\
Garganta seca x comparação temporal da intensidade vocal no fortíssimo & 5,99 & 2,83 \\
Garganta seca x comparação temporal da qualidade vocal & 5,99 & 2,16 \\
Garganta seca x comparação temporal da passagem & 5,99 & 0,89 \\
Garganta seca x comparação temporal da afinação & 5,99 & 4,11 \\
Garganta seca x fala em intensidade forte & 5,99 & 2,45 \\
Garganta seca x comer tarde da noite & 5,99 & 0,30 \\
Acúmulo de secreção na garganta x voz cantada eficiente & 3,84 & 2,37 \\
Acúmulo de secreção na garganta x comparação temporal da tessitura vocal & 5,99 & 1,37 \\
Acúmulo de secreção na garganta x comparação temporal da intensidade no pianíssimo & 5,99 & $8,90^{\star}$ \\
Acúmulo de secreção na garganta x comparação temporal da intensidade no fortíssimo & 5,99 & 0,99 \\
Acúmulo de secreção na garganta x comparação temporal da qualidade vocal & 5,99 & $7,40^{*}$ \\
Acúmulo de secreção na garganta x comparação temporal da passagem & 5,99 & $8,19^{*}$ \\
\hline
\end{tabular}

TESTE Qui-quadrado

Acúmulo de secreção na garganta $x$ voz falada eficiente

Teste: Fischer $\mathrm{P}=0,0163^{*}$

Acúmulo de secreção na garganta está associado a voz falada menos eficiente 
Tabela 9 - Análise comparativa do grau de relacionamento entre as queixas de cansaço após fala e cansaço após canto e parâmetros de voz falada e cantada

\begin{tabular}{lcc}
\hline Parâmetros & $\begin{array}{c}\text { Valor } \\
\text { crítico }\end{array}$ & $\begin{array}{c}\text { Valor } \\
\text { observado }\end{array}$ \\
\hline Cansaço após fala x ensaio dos naipes em separado & 3,84 & 1,40 \\
Cansaço após fala x comparação temporal da tessitura vocal & 5,99 & 3,12 \\
Cansaço após fala x comparação temporal da intensidade no pianíssimo & 5,99 & 0,83 \\
Cansaço após fala x comparação temporal da intensidade no fortíssimo & 5,99 & $6,01^{*}$ \\
Cansaço após fala x comparação temporal da qualidade vocal & 5,99 & 5,32 \\
Cansaço após fala com relação a passagem & 5,99 & 4,17 \\
Cansaço após fala x falar em intensidade forte & 5,99 & 0,82 \\
Cansaço após fala x comer tarde da noite & 5,99 & 1,73 \\
Cansaço após canto x ensaio de naipes em separado & 3,84 & $3,90^{*}$ \\
Cansaço após canto x voz falada em relação a cantada & 5,99 & $9,09^{*}$ \\
Cansaço após canto x eficiência da voz cantada & 3,84 & $7,47^{*}$ \\
Cansaço após canto x comparação temporal da tessitura vocal & 5,99 & 4,36 \\
Cansaço após canto x compaçãão temporal da intensidade no pianíssimo & 5,99 & 2,89 \\
Cansaço após canto x comparação temporal da intensidade no fortíssimo & 5,99 & 5,41 \\
Cansaço após canto x compaçãão temporal da qualidade vocal & 5,99 & 5,30 \\
Cansaço após canto x compaçãão temporal da passagem & 5,99 & 2,41 \\
Cansaço após canto x comparação temporal da afinação & 5,99 & 2,05 \\
Cansaço após canto x fala em intensidade forte & 5,99 & 3,10 \\
Cansaço após canto x comer tarde da noite & 5,99 & 0,29 \\
\hline
\end{tabular}

TESTE Qui-quadrado

Tabela 10 - Análise comparativa do grau de relacionamento entre a queixas de tensão na garganta e parâmetros de voz falada e cantada

\begin{tabular}{lcc}
\hline Parâmetros & $\begin{array}{c}\text { Valor } \\
\text { crítico }\end{array}$ & $\begin{array}{c}\text { Valor } \\
\text { observado }\end{array}$ \\
\hline Tensão na garganta x ensaio de naipes em separado & 3,84 & 0,60 \\
Tensão na garganta x voz falada em relação a cantada & 5,99 & 1,31 \\
Tensão na garganta x comparação temporal da tessitura vocal & 5,99 & 1,85 \\
Tensão na garganta x comparação temporal da intensidade no pianíssimo & 5,99 & $8,27^{\star}$ \\
Tensão na garganta x comparação temporal da intensidade no fortíssimo & 5,99 & 0,40 \\
Tensão na garganta x comparação temporal da qualidade vocal & 5,99 & $9,01^{*}$ \\
Tensão na garganta x comparação temporal da passagem & 5,99 & 0,40 \\
Tensão na garganta x comparação temporal da afinação & 5,99 & 4,06 \\
Tensão na garganta x fala em intensidade forte & 5,99 & 0,60 \\
Tensão na garganta x comer tarde da noite & 5,99 & 1,01 \\
\hline
\end{tabular}

TESTE Qui-quadrado

possível observar que: 31 sujeitos $(20,7 \%)$ não apresentaram nenhum sintoma, 86 sujeitos $(57,3 \%)$ apresentaram até 3 sintomas, 28 sujeitos $(18,7 \%)$ apresentaram até 6 sintomas e 5 sujeitos (3,3\%) apresentaram mais de 6 sintomas (Tabela 11).

Com relação aos hábitos vocais constata-se que: 137 (91,3\%) regentes não fumam, 105 (70\%) não bebem, $92(61,3 \%)$ não gritam e70 $(46,7 \%)$ não pigarreiam; 90 (60\%) falam muito e 65 (43,3\%) comem tarde da noite. $O$ ítem relacionado a falar em intensidade forte ficou equilibrado entre sim $(36,0 \%)$ e não $(35,3 \%)$ (Tabela 12$)$.
Tabela 11 - Distribuição numérica e percentual do número de sintomas entre os regentes

\begin{tabular}{lcc}
\hline \multirow{2}{*}{ № de sintomas } & \multicolumn{2}{c}{ Regentes } \\
\cline { 2 - 3 } & $\mathbf{n}$ & $\%$ \\
\hline 0 & 31 & 20,7 \\
$1 \longmapsto 3$ & 86 & 57,3 \\
$4-6$ & 28 & 18,7 \\
$6 \vdash$ & 5 & 3,3 \\
\hline
\end{tabular}


Tabela 12 - Distribuição numérica e percentual dos hábitos dos regentes

\begin{tabular}{lcccccc}
\hline \multirow{2}{*}{ Hábito } & \multicolumn{2}{c}{ Sim } & \multicolumn{2}{c}{ Não } & \multicolumn{2}{c}{ Às vezes } \\
\cline { 2 - 7 } & $\mathbf{n}$ & $\%$ & $\mathbf{n}$ & $\%$ & $\mathbf{n}$ & $\%$ \\
\hline Fumar & 5 & 3,3 & 137 & 91,3 & 8 & 5,3 \\
Beber & 5 & 3,3 & 105 & 70,0 & 40 & 26,7 \\
Falar muito & 90 & 60,0 & 25 & 16,7 & 35 & 23,3 \\
Falar em intensidade forte & 54 & 36,0 & 53 & 35,3 & 43 & 28,7 \\
Gritar & 14 & 9,3 & 92 & 61,3 & 44 & 29,3 \\
Pigarrear & 17 & 11,3 & 70 & 46,7 & 63 & 42,0 \\
Comer tarde da noite & 65 & 43,3 & 27 & 18,0 & 58 & 38,7 \\
\hline
\end{tabular}

\section{DISCUSSÃo}

A fim de traçar o perfil vocal de regentes de coral do Estado de São Paulo, foram questionados e analisados diversos aspectos particulares referentes a esta atividade profissional.

A maior parte dos regentes estudados, faz ou já fez aulas de canto em média de um a cinco anos. A prática de aulas de canto é elencada por muitos autores como um aprimoramento essencial para cantores e regentes de coral e devem, de preferência, durar anos ${ }^{8,10}$. Com aulas de canto, o profissional desenvolve potencialmente todo seu mecanismo vocal, o que propicia uma melhor qualidade de voz cantada sem esforço. Alguns autores comentam ainda que aulas de canto também desenvolvem o controle da passagem e da intensidade da voz ${ }^{10,11}$.

Com relação à classificação vocal dos regentes, verificou-se que entre as vozes femininas, a de menor ocorrência foi a de contralto 19 regentes, e entre as masculinas, a de menor ocorrência foi a de baixo 6 regentes. Estes dados vêm ao encontro da literatura que aponta as vozes de contralto e baixo como sendo raras ${ }^{8,11}$.

A prática de ensaio de naipes em separado e a de cantar junto com os naipes são apontadas na literatura específica para o exercício da regência como facilitadoras no ensaio de peças mais complexas, e como uma forma de o regente dominar completamente as dificuldades rítmicas, intervalares, de respiração e de emissão para poder transmitir ao coro as nuances da música ${ }^{1,12}$. Ao praticarem, em sua maioria o aquecimento vocal, os regentes se colocam em concordância com a da literatura pesquisada, que sugere este procedimento como fundamental antes de ensaios e apresentações como medida funcional, profilática e mantenedora de uma boa voz cantada ${ }^{8,11,13-17}$. Com relação ao desaquecimento vocal, observou-se que os regentes, em sua maioria não o praticam. O desaquecimento vocal é citado na literatura mais recente como um meio que propicia o retorno do ajuste motor da voz cantada para a falada, evitando desgaste e aumentando a longevidade vocal ${ }^{14}$. O fato de a maioria dos regentes não praticarem o desaquecimento vocal pode indicar a falta de conhecimento de seus benefícios.

Utilizar o piano e a própria voz como modelo na afinação no ensaio de coristas que compõem os naipes faz parte da pedagogia utilizada pelos regentes ${ }^{2-4,7}$. Espera-se que o regente tenha plasticidade vocal, que seja capaz de praticar a variabilidade de freqüência e intensidade, e que tenha domínio vocal do canto musical ${ }^{18}$, porém a prática de canto em diversos naipes pode representar um risco para o desenvolvimento de alterações laríngeas ${ }^{8,12,19}$.

Muitos cantores se beneficiam com treinamento específico para a voz falada, da mesma maneira que muitos falantes se beneficiam do treinamento específico para a voz cantada. Cantores não treinados normalmente falam mais abusivamente do que cantam ${ }^{6,20}$.

Na questão referente ao componete básico para uma boa voz cantada, dom e/ou técnica, obtevese dom e técnica na maioria das respostas. Estes dados corroboram com a literatura que aponta estes dois aspectos como complementares quando se trata de uma boa voz cantada ${ }^{11,17}$.

Os aspectos relacionados a tessitura, qualidade vocal, passagem e afinação são apontados na literatura como diretamente relacionados a prática contínua da voz cantada ${ }^{5}$. Na mesma literatura encontrou-se que as dificuldades específicas concernentes as zonas de passagem dependem em especial de treino minucioso para que possam ser superadas pelo cantor ${ }^{8,11}$. $\mathrm{O}$ fato de a intensidade no pianíssimo e no fortíssimo terem apresentado um alto índice de inalteração, poderia indicar a falta de preocupação do regente com estes aspectos específicos, uma vez que o controle fino da intensidade no canto geralmente é exigido para cantores solistas. 
As queixas vocais mais apontadas pelos regentes, em ordem decrescente foram: pigarro, rouquidão, garganta seca, acúmulo de secreção na garganta, cansaço após fala, cansaço após canto e tensão na garganta.

O pigarro está relacionado ao aumento da viscosidade do muco da região laríngea sendo apontado na literatura como indicador de falta de hidratação e higiene vocal pobre ${ }^{8,9,21}$. A literatura é concordante quando se refere ao hábito de pigarrear como um dos fatores de maior abuso vocal, sendo enfática ao aconselhar aos cantores que evitem ao máximo este hábito ${ }^{8,11}$. Quando se compara pigarro a eficiência das vozes falada e cantada e a especificidades para a voz cantada, não se observa significância estatística.

A rouquidão é considerada como sintoma clássico de alteração e/ou produção vocal anormal, podendo ser conseqüência desde um leve edema até alterações importantes de massa das pregas vocais ${ }^{22,23}$. Quando se compara rouquidão a especificidades da voz cantada, observa-se que a rouquidão está associada a uma tessitura mais restrita, a uma pior intensidade no pianíssimo, a qualidade vocal menos estável e a uma pior passagem, o que leva a observar que a rouquidão é um dos sintomas que mais se relacionam a dificuldades na produção da voz cantada.

A garganta seca é um sintoma freqüente entre os profissionais da voz, podendo provocar dificuldades à emissão ${ }^{8,9,21}$. Ao comparar a queixa de garganta seca e especificidades da voz cantada, encontra-se sua associação a uma tessitura vocal mais restrita. A perda de notas dos extremos da tessitura, grave e agudo, pode ser o primeiro sintoma de distúrbios vocais importantes ${ }^{8}$.

Ao comparar acúmulo de secreção na garganta a especificidades para a voz cantada observa-se que o mesmo está associado a voz falada menos eficiente, a pior intensidade no pianíssimo, a qualidade vocal menos estável e a uma pior passagem. O acúmulo de secreção na garganta, embora citado por apenas 37 dos estrevistados, mostra, como a rouquidão, como um dos sintomas que mais se relacionam a dificuldades na produção da voz cantada $8,9,21$.

Cansaço após fala e cansaço após canto relacionam-se à fadiga vocal conseqüente de uso excessivo das vozes falada e cantada ${ }^{21,24-26}$. O cansaço após fala quando comparado a outras características aparece associado a uma pior intensidade no fortíssimo; o cansaço após canto por sua vez, está associado ao ensaio de naipes em separado, a uma voz falada melhor que a cantada e a uma voz cantada não eficiente.
A tensão na garganta pode estar relacionada a um uso ou esforço excessivo na produção vocal, sendo considerado sinal de mau uso ${ }^{27}$. Quando se compara o sintoma de tensão na garganta a especificidades vocais para o canto observa-se que a mesma está associada a uma pior qualidade no pianíssimo e a uma qualidade vocal menos estável.

A literatura aponta que na presença de sintomas vocais múltiplos, 3 ou mais podem indicar severidade de atrito vocal. Na presente mostra, somente $22 \%$ se encaixam nesta categoria ${ }^{28}$. Nessa amostra o parâmetro que mais se alterou frente aos sintomas vocais foi a qualidade vocal, o que pode constituir um fator de risco para o desenvolvimento de alterações vocais ocupacionais ${ }^{29-31}$.

A literatura mostra-se concordante com relação a extrema nocividade dos hábitos de fumar e beber para a saúde geral e vocal hábitos que a maior parte dos regentes refere não possuir. O hábito de comer tarde da noite, apontado por $43,3 \%$ dos regentes, pode favorecer o refluxo-gastro esofágico ${ }^{33}$.

As queixas apresentadas pelos regentes de coral, bem como os hábitos nocivos, poderiam ser minimizados com a atuação do Fonoaudiólogo na higiene vocal e no controle de abuso e mau uso vocal na voz falada e cantada ${ }^{33,34}$.

\section{CONCLUSÕES}

Com base nos achados deste estudo pode-se concluir que o perfil vocal de regentes de coral do Estado de São Paulo engloba as seguintes características:

- Os regentes tem em média 8,4 anos de exercício na profissão, regem um coral e já fizeram de um a cinco anos de aulas de canto. Em sua maioria, ensaiam os naipes em separado, cantam junto com os naipes, fazem aquecimento vocal, não fazem desaquecimento vocal e usam para a afinação do coral, piano e/ou a própria voz. A maioria considera a qualidade da voz falada igual a cantada e ambas eficientes. Houve concordância no que se referiu ao dom e a técnica como componentes básicos para uma boa voz cantada.

- Com os anos de exercício profissional os regentes referem que a tessitura vocal está mais ampla, a qualidade mais estável, a passagem mais controlada e a afinação melhor. A intensidade no pianíssimo e no fortíssimo se mostraram entre melhor e inalterada.

- As queixas vocais mais apontadas foram: pigarro, rouquidão, garganta seca, acúmulo de secreção na garganta, cansaço após fala, cansaço após canto e tensão na garganta. 
- A rouquidão está associada a tessitura mais restrita, pior intensidade no pianíssimo, qualidade vocal menos estável e passagem pior. $\mathrm{O}$ acúmulo de secreção na garganta, por sua vez, está associado a uma voz falada não eficiente, pior intensidade no pianíssimo, qualidade vocal menos estável e a uma pior passagem. O cansaço após fala está associado a uma pior intensidade no fortíssimo e o cansaço após canto ao ensaio de naipes em separado, a uma voz falada melhor que a cantada, a uma voz cantada não eficiente, a pior intensidade no pianíssimo e a qualidade vocal menos estável. A tensão na garganta está associada a uma voz falada menos eficiente, a pior intensidade no pianíssimo e a uma qualidade vocal menos estável. A garganta seca a uma tessitura mais restrita.

- Os regentes em sua maioria não fumam, não bebem, não costumam gritar, não pigarreiam, falam muito e comem tarde da noite. A maior parte apresenta no máximo três sintomas vocais. O parâmetro que mais se alterou frente aos sintomas vocais foi a qualidade vocal.

\section{ABSTRACT}

Purpose: to draw the vocal profile of choir conductors in the State of São Paulo. Methods: one hundred fifty choirs conductors in the State of São Paulo took part in this study. Data collection was carried out by the same researcher, at the working places of the subjects by means of closed survey. Results: the conductor's average performance of the activity is 8.4 years. They conduct different sections at different times, they sing together with the sections, do warming up but do not do cooling downs. For choir tune up they use the piano and/or their own voice. Most of them consider spoken voice similar to sung voices and both are efficient. There is agreement concerning talent and technique as necessary components for a good sung voice. After having been conductors for years, the vocal range is broader, the vocal quality is more stable and passage more controlled. The intensity at pianissimo and fortissimo remains unaltered. The vocal complains that are most observed were hawks, hoarseness, dry throat, excess of secretion in the throat, fatigue after singing and tension in the throat. Most conductors complained about up to three vocal symptoms, do not smoke, do not drink, do not usually scream, do not hawk, speak a lot and eat late at night. Conclusion: vocal symptoms were associated with worsening and/or restriction of several specific parameters of singing voice. The most altered parameter among the symptoms was the vocal quality.

KEYWORDS: Voice; Voice Quality; Voice Training; Music; Voice Disorders

\section{REFERÊNCIAS}

1. Zander O. Regência coral. 5. ed. Porto Alegre: Movimento; 2003. 89p.

2. Fernades AJ, Kayama AG, Östergren EA. O regente moderno e a construção da sonoridade coral. Per Musi. 2006; 13:33-51.

3. Ginsborg J, Chaffin R, Nicholson G. Shared performance cues in singing and conducting: a content analysis of talk during practice. Psychol Music. 2006; 34(2):167-94.

4. Durrant C. Less is more: conducting gesture. In: Durrant C. Choral conducting: philosophy and practice. New York: Routledge; 2003. p.136-52.

5 . Sundberg J. Research on the singing voice in restrospect. TMH-QPSR. 2003; 45(1):11-22.

6. Rehder MIBC. Análise vocal, perceptivo-auditiva e acústica, falada e cantada de regentes de coral [doutorado]. São Paulo (SP): Universidade Federal de São Paulo; 2002.

7. Fernades AJ, Kayama AG, Östergren EA. A prática coral na atualidade: sonoridade, interpretação e técnica vocal. Musica Hodie. 2001; 6(1):51-74.

8. Behlau MS, Rehder MIBC. Higiene vocal para o canto coral. São Paulo: Revinter; 1997. 44p.

9. Behlau MS, Feijó D, Madazio G, Rehder MI, Azevedo R, Ferreira AE. Voz profissional: aspectos gerais e atuação fonoaudiológica. In: Behlau MS. Voz: o livro do especialista. v. 2. Rio de Janeiro: Revinter; 2005. p.287-406.

10. Watts C, Barnes-Burroughs K, Estis J, Blanton $D$. The singing power ratio as an objective measure of singing voice quality in untrained talented and nontalented singers. J Voice. 2006; 20(1):82-8.

11. Silva MA, Duprat AC. Voz cantada. In: Ferreira LP, Befi-Lopes DM, Limongi SCO. Tratado 
de fonoaudiologia. São Paulo: Rocca; 2004. p.177-194.

12. Pela S, Rehder MI, Behlau MS. O trabalho fonoaudiológico com corais. In: Marchesan IQ, Zorzi JL, Gomes ICD. Tópicos em fonoaudiologia 1997/1998. São Paulo: Lovise; 1998. p. 529-43.

13. Titze I. Choir warms-ups: how effective are they? J Sing. 2000; 56:31-2.

14. Scarpel RD, Pinho SMR. Aquecimento e desaquecimento vocal. In: Pinho SMR, organizador. Tópicos de voz. Rio de Janeiro: Guanabara Koogan; 2002.

15. Amir O, Amir N, Michaeli $\mathrm{O}$. Evaluating the influence of warmup on singing voice quality using acoustic measures. J Voice. 2005; 19(2):252-60.

16. Milbrath RL, Solomon NP. Do vocal warm-up excercises alleviate vocal fatigue? J Speech Lang Hear Res. 2003; 46(2):422-36.

17. Andrade SR, Cielo CA. A ciência fonoaudiológica e a arte do canto coral. Fono Atual. 2005; 8(33):59-68.

18. Behlau MS. Vozes preferidas: considerações sobre opções vocais nas profissões. Fono Atual. 2001; 4:10-4.

19. Vasilenko L, Pavlikhin OG, Izgarysheva ZA. Analysis of the causes of vocal tract diseases in singers. Vestn Otorinolaringol. 2000; 5:13-7.

20. Pillot $\mathrm{C}$, Vaissière $\mathrm{J}$. Vocal effectiveness in speech and singing: acoustical, physiological and perceptive aspects. Applications in speech therapy. Rev Laryngol Otol Rhinol (Bord). 2006; 127(5):293-8.

21. Solomon NP, Glaze LE, Arnold RR, Van Mersbergen M. Effects of a vocally fatiguing task and systemic hydration on men's voices. J Voice. 2003; 17(1):31-46.

22. Banfield G, Tandon P, Solomons N. Hoarse voice: an early symptom of many conditions. Practitioner. 2000; 244(1608):267-71.

23. Halberstam B. Acoustic and perceptual parameters relating to connected speech are more reliable measures of hoarseness than parameters relating to sustained vowels. ORL J Otorhinolaryngol Relat Spec. 2004; 66(2):70-3.

24. Emerich K, Sataloff RT. Chronic fatigue syndrom singers. In: Sataloff RT. Professional voice: the science and art of clinical care. San Diego: Singular; 1997 p.447-51.

25. Welham NV, Maclagan MA. Vocal fatigue: current knowledge and future directions. J Voice. 2003; 17(1):21-30.

26. Estienne F. Voz falada, voz cantada. Rio de Janeiro: Revinter; 2004. 64p.

27. Davies J, Anderson S, Huchison L, Stewart $\mathrm{G}$. Interactions between voice clinics and singing teachers: a report on the British Voice Association questionnaire to voice clinics in the UK. Logoped Phoniatr Vocol. 2007; 32(2):83-6.

28. Nagano L, Dragone MLS, Behlau MS. Estudos sobre a relação entre qualidade vocal e sintomas vocais. In: Marchesan IQ, Zorzi JL. Anuário do CEFAC 1999/2000. Rio de Janeiro: Revinter; 2000. p. 293-303.

29. Sataloff RT. Professional voice users: the evaluation of voice disorders. Occup Med. 2001; 16(4):633-47.

30. Timmermans B, De Bodt MS, Wuyts FL, Boudewijns A, Clement G, Peeters A, Van de Heyning $\mathrm{PH}$. Poor voice quality in future elite vocal performers and professional voice users. J Voice. 2002; 16(3):372-82.

31. Schneider B, Cecon M, Hanke G, Wehner S, Bigenzahn W. [Significance of voice constitution as a predisposition for occupational voice disorders]. HNO. 2004; 52(5):461-7.

32. Cammarota G, Masala G, Cianci R, Palli D, Capaccio P, Schindler A, et al. Reflux symptoms in professional opera choristers. Gastroenterol. 2007; 132(3):890-8.

33. Gutiérrez CA. La voz cantada: interaccion del fonoaudiologo con el cantante. Acta Otorrinolaringol Cir Cabeza Cuello. 2003; 31(2Supl):57-60.

34. Timmermans B, Vanderwegen J, De Bodt MS. Outcome of vocal hygiene in singers. Curr Opin Otolaryngol Head Neck Surg. 2005; 13(3):138-42.

RECEBIDO EM: 08/05/2008

ACEITO EM: 15/06/2008

Endereço para correspondência:

Av. 15,531

Rio Claro - SP

CEP: 13500330

Tel: (19) 3524-5321 / (19) 8111-5169

E-mail: mariainesrehder@uol.com.br 